\title{
Influence of paclobutrazol on growth performance, photosynthetic pigments, and antioxidant efficiency of Pathumthani 1 rice seedlings grown under salt stress
}

\author{
Bundit Khunpon $^{\mathrm{a}}$, Suriyan Cha-um ${ }^{\mathrm{b}}$, Bualuang Faiyue ${ }^{\mathrm{c}}$, Jamnong Uthaibutra ${ }^{\mathrm{a}}$, Kobkiat Saengnil ${ }^{\mathrm{a}, *}$ \\ a Department of Biology, Faculty of Science, Chiang Mai University, Chiang Mai 50200 Thailand \\ b National Centre for Genetic Engineering and Biotechnology (BIOTEC), \\ National Science and Technology Development Agency, 113 Thailand Science Park, Pahonyothin Road, \\ Khlong Nueng, Khlong Luang, Pathum Thani 12120 Thailand \\ c Department of Biology, Mahidol Wittayanusorn School, Salaya, Phuttamonthon, \\ Nakhon Pathom 73170 Thailand \\ *Corresponding author, e-mail: kobkiat_s@hotmail.com
}

Received 9 Jun 2016

Accepted 25 Mar 2017

\begin{abstract}
The ability of paclobutrazol to ameliorate salinity stress, growth performance, photosynthetic pigment, and antioxidant efficiency was studied in indica rice cv. Pathumthani 1. Fourteen-day-old rice seedlings were pretreated with $15 \mathrm{mg} / \mathrm{l}$ paclobutrazol by foliar spray and subsequently exposed to salt stress (150 mM NaCl) for 12 days. Salt stress decreased growth characters including fresh weight, dry weight, shoot length, root length, shoot fresh weight, shoot dry weight, root fresh weight, and root dry weight. It also reduced the photosynthetic pigments chlorophyll $\mathrm{a}$, chlorophyll b, total chlorophyll, and total carotenoids. In addition, salinity stress decreased the total antioxidant capacity as determined by 2,2-diphenyl-1-picrylhydrazyl, 3-ethylbenzothiazoline-6-sulphonic acid, and ferric-reducing antioxidant power assays and reduced the activities of the antioxidant enzymes, peroxidase and polyphenol oxidase, in both leaves and roots. Seedling growth retardation and pigment degradation were positively correlated with the reduction of antioxidant efficiency under salt stress. Paclobutrazol pretreatment improved growth, pigment production, and antioxidant efficiency in salt-stressed rice seedlings. These results indicate that paclobutrazol plays an important role in ameliorating the impact of salinity stress on Pathumthani 1 rice seedlings.
\end{abstract}

KEYWORDS: chlorophyll, enzymatic antioxidant, antioxidant capacity, salinity stress, Oryza sativa

\section{INTRODUCTION}

Soil salinity is a major environmental problem in agricultural areas. The total area of saline and sodic soils in the world covers approximately 800 million ha ${ }^{1}$. The effects of salinity on plants are caused by the osmotic effect when high concentrations of salt are accumulated in soil, lowering water potential and reducing water uptake by the root. It is also caused by ionic effect when the salt transported to the shoot exceeds the capacity of cells, disrupting physiological and biochemical processes $^{2}$.

Salinity reduces photosynthetic rate and photosynthetic pigments leading to a reduction in the plant growth and survival ${ }^{3,4}$. Many studies have also confirmed that reduction in the photosynthetic pigments such as chlorophylls and carotenoids in plant grown under salinity mainly contributes to the decline in photosynthesis ${ }^{3,5}$.

Salt stress also induces the accumulation of reactive oxygen species (ROS) such as superoxide radical $\left(\mathrm{O}_{2}{ }^{--}\right)$, hydrogen peroxide $\left(\mathrm{H}_{2} \mathrm{O}_{2}\right)$, and hydroxyl radical ( $\mathrm{HO}^{\circ}$ ), causing oxidative damages of lipids, proteins, and chlorophylls ${ }^{6}$. Plants possess an antioxidant defence systems including enzymatic antioxidants such as superoxide dismutase (SOD), catalase (CAT), ascorbate peroxidase (APX), glutathione reductase, peroxidase (POX), and polyphenol oxidase (PPO), and non-enzymatic antioxidants such as ascorbate, glutathione, and $\alpha$-tocopherol ${ }^{7}$. Consequently, an increase in antioxidant efficiency contributes to the protection of plants against salt stress $^{6,8}$. 
The enhancement of salt tolerance in plants can be achieved through exogenous application of plant growth regulators such as salicylic acid and polyamines ${ }^{9,10}$. Paclobutrazol (PBZ) [(2RS,3RS)1-(4-chlorophenyl)-4,4-dimethyl-2-(1,2,4-triazol-1yl)pentan-3-ol] is a triazole fungicide which regulates the plant growth by antagonizing the hormone gibberellin. Triazole interferes with gibberellin biosynthesis by inhibiting the ent-kaurene oxidase enzyme which catalyses the oxidation of ent-kaurene to ent-kaurenoic acid in the terpenoid pathway for the production of gibberellins through inactivating cytochrome $\mathrm{P}_{450}$-dependent monooxygenases ${ }^{11}$. In general, PBZ and triazole compounds cause many morphological and physiological responses in plants such as stimulating root growth, reducing shoot growth ${ }^{12,13}$, increasing chlorophyll content, promoting net photosynthetic rate, ameliorating carbohydrate content ${ }^{11}$, reducing free-radical damage, enhancing antioxidant efficiency ${ }^{14}$, regulating cytokinin biosynthesis, and inhibiting abscisic acid biosynthesis ${ }^{11}$.

Recent studies have shown beneficial effects of PBZ against abiotic stresses such as flooding ${ }^{15}$, drought ${ }^{14}$, and salinity ${ }^{12}$. The protection mechanisms against abiotic stresses using exogenous PBZ treatment are linked to antioxidant defence systems such as SOD, CAT, APX, and POX enzyme activities as well as ascorbate, glutathione, and $\alpha$-tocopherol non-enzymatic enrichment ${ }^{12-14}$.

In Thailand, Pathumthani 1 (PTT1) rice, a photoperiod-insensitive cultivar, is the most popular variety in irrigated paddy field because it produces a high yield, long grain, good cooking quality, and three crop rotations per annum. However, PTT1 has been well-categorized as being salt-susceptible in both seedling and reproductive stages, reducing the productivity ${ }^{16}$. Although PBZ has been shown to alleviate stress injury in several plants, the effect of PBZ on growth, photosynthetic pigments, and antioxidant efficiency in PTT1 rice under salinity has not been reported. In this study, it was hypothesized that PBZ may enhance antioxidant efficiency that prevents degradation of photosynthetic pigments and promote growth of PTT1 rice seedlings under a salt stress condition.

\section{MATERIALS AND METHODS}

\section{Plant materials}

Seeds of rice cv. Pathumthani1 (PTT1) obtained from the Pathumthani Rice Research Centre, Rice Research Institute, Department of Agriculture, Min- istry of Agriculture and Cooperative, Thailand, were soaked in distilled water for $18 \mathrm{~h}$. The seeds were then placed in a plastic tray $(20 \mathrm{~cm} \times 30 \mathrm{~cm})$ covered with moist tissue papers for germination in the dark at room temperature $\left(25 \pm 1^{\circ} \mathrm{C}\right)$ for $72 \mathrm{~h}$. Germinated seedlings were taken from the tray and 10 seedlings were planted in each plastic pot (30 $\mathrm{cm}$ in diameter and $30 \mathrm{~cm}$ in height) filled with $3 \mathrm{~kg}$ of clay soil $(\mathrm{EC}=1.787 \mathrm{dS} / \mathrm{m} ; \mathrm{pH}=6.42$; organic matter $=7 \%$; total nitrogen $=0.26 \%$; total phosphorus $=0.05 \%$; total potassium $=1 \%$ ) and grown for 14 days under $70 \pm 5 \%$ relative humidity with an average temperature of $26 \pm 6{ }^{\circ} \mathrm{C}$, and 500-1000 $\mu \mathrm{mol} \mathrm{m}{ }^{-2} \mathrm{~s}^{-1}$ photosynthetic photon flux density with $10 \mathrm{~h} / \mathrm{d}$ photoperiod.

\section{PBZ pretreatment and salt stress}

Fourteen-day-old rice seedlings grown in pots were applied with distilled water (48 pots) or $15 \mathrm{mg} / 1$ PBZ (48 pots) by a foliar spray using a handheld sprayer at $50 \mathrm{ml} /$ pot. After 7 days, 24 pots of control and 24 pots of PBZ treatment were put in 15-1 plastic buckets (one pot/bucket) filled with tap water, whereas other 24 pots of control and 24 pots of PBZ treatment were put in 15-1 plastic buckets filled with $150 \mathrm{mM} \mathrm{NaCl}$.

The buckets were arranged in a completely randomized design with three replicates for each treatment. Rice seedlings were randomly harvested at -7 ( 7 days before salt exposure started), 0,1 , $3,5,7,9$, and 12 days from salinization to analyse growth performances, photosynthetic pigments and antioxidant efficiencies.

\section{Measurement of growth performances}

Shoot and root of rice seedlings were gently separated and weighed for shoot fresh weight (SFW), and root fresh weight (RFW). Shoot height (SH) and root length (RL) were measured with a ruler. Fresh samples were then dried at $80^{\circ} \mathrm{C}$ in a hot air oven for 3 days and then shoot dry weight (SDW) and root dry weight (RDW) were recorded.

\section{Analysis of the photosynthetic pigments}

Chlorophyll a $\left(\mathrm{Chl}_{\mathrm{a}}\right)$, chlorophyll b $\left(\mathrm{Chl}_{\mathrm{b}}\right)$, total chlorophyll (TC) and total carotenoid $\left(\mathrm{C}_{\mathrm{x}+\mathrm{c}}\right)$ concentrations were determined according to the method of Arnon ${ }^{17}$ with some modifications. Twenty milligrams of leaf tissues were finely sliced and placed in a test tube containing $5 \mathrm{ml}$ of $80 \%$ acetone. The tube was placed in a dark room at $25 \pm 1{ }^{\circ} \mathrm{C}$ for 1 day. Absorbance of the extracted 
solution was measured with a visible spectrophotometer (Thermo model Genesis 20, USA) at 470, 645 , and $663 \mathrm{~nm}$. A solution of $80 \%$ acetone was used as blank.

\section{Determination of total antioxidant capacities (TAC)}

Two hundred milligrams of rice leaves or roots were sliced and homogenized in $10 \mathrm{ml}$ of $80 \%$ methanol for $1 \mathrm{~min}$ at $4^{\circ} \mathrm{C}$. The homogenate was centrifuged at $16000 \mathrm{~g}$ (Hermel model Z383K, Germany), $4^{\circ} \mathrm{C}$ for $20 \mathrm{~min}$. The supernatant was collected to determine the TAC by using $2,2^{\prime}$ azino-bis(3-ethylbenzothiazoline-6-sulphonic acid) (ABTS), 2,2-diphenyl-1-picrylhydrazyl (DPPH) radical scavenging, and ferric-reducing antioxidant power (FRAP) assays.

ABTS radical scavenging activity was assayed based on the modified method of Huang et $\mathrm{al}^{18}$. $\mathrm{ABTS}^{+}$solution was prepared by adding $2 \mathrm{ml}$ of $7 \mathrm{mM}$ ABTS to $3 \mathrm{ml}$ of $2.45 \mathrm{mM}$ potassium persulphate. The mixture was kept in the dark at $5{ }^{\circ} \mathrm{C}$ for $16 \mathrm{~h}$ to give a dark blue solution before being diluted with $80 \%$ ethanol until the absorbance was read as 0.7 at $734 \mathrm{~nm}$. Then $0.02 \mathrm{ml}$ of the supernatant was mixed with $2 \mathrm{ml}$ of $\mathrm{ABTS}^{+}$solution. The mixture was stored at room temperature for $10 \mathrm{~min}$ and the absorbance was measured at $734 \mathrm{~nm}$. ABTS radical scavenging activity was compared with that of Trolox, used as a water-soluble vitamin E analogue. TAC was determined and expressed as $\mu \mathrm{mol}$ Trolox/g FW (fresh weight).

DPPH radical scavenging activity was determined according to the modified method of Mun'im et $\mathrm{al}^{19}$. The supernatant of $0.1 \mathrm{ml}$ was mixed with $0.4 \mathrm{ml}$ of $0.3 \mathrm{M}$ acetate buffer $(\mathrm{pH} \mathrm{5.5)}$ and $2.5 \mathrm{ml}$ of $0.12 \mathrm{mM} \mathrm{DPPH}^{*}$ with $100 \%$ methanol. The mixture was kept in the dark at room temperature $\left(25 \pm 1{ }^{\circ} \mathrm{C}\right)$ for $30 \mathrm{~min}$ and the absorbance was measured at $517 \mathrm{~nm}$. DPPH radical scavenging activity was compared with that of Trolox. TAC was determined and expressed as $\mu \mathrm{mol}$ Trolox/g FW.

FRAP assay was performed based on the modified methods of Benzie and Strain ${ }^{20}$. FRAP solution was freshly prepared by mixing $300 \mathrm{mM}$ acetate buffer (pH 3.6), $10 \mathrm{mM} \mathrm{2,4,6-tripyridyl-s-}$ triazine in $40 \mathrm{mM} \mathrm{HCl}$ and $20 \mathrm{mM} \mathrm{FeCl}_{3} \cdot 6 \mathrm{H}_{2} \mathrm{O}$ $\left(\mathrm{FeCl}_{3} \cdot 6 \mathrm{H}_{2} \mathrm{O}\right)$ at the ratio of 10:1:1. Then $0.3 \mathrm{ml}$ of the supernatant was combined with $2.7 \mathrm{ml}$ of FRAP solution. The mixture was placed in the dark at room temperature $\left(25 \pm 1^{\circ} \mathrm{C}\right)$ for $30 \mathrm{~min}$ and the absorbance was measured at $593 \mathrm{~nm}$. FRAP was compared with $\mathrm{FeSO}_{4}$. TAC was determined and expressed as $\mu \mathrm{mol} \mathrm{Fe}^{2+} / \mathrm{g}$ FW.

\section{Determination of enzymatic antioxidant activities}

POX and PPO were extracted by using the method of Huang et $\mathrm{al}^{21}$ with some modifications. Two hundred milligrams of rice leaf or root tissues were sliced and homogenized in $20 \mathrm{ml}$ of $0.05 \mathrm{M}$ $\mathrm{K}_{3} \mathrm{PO}_{4}$ buffer ( $\mathrm{pH}$ 6.2) containing $1 \mathrm{M} \mathrm{KCl}$ and $2 \%$ polyvinylpyrrolidone for $1 \mathrm{~min}$ at $4^{\circ} \mathrm{C}$. The homogenate was centrifuged at $20000 \mathrm{~g}$ (Hermel model Z383K, Germany), $4{ }^{\circ} \mathrm{C}$ for $5 \mathrm{~min}$. The supernatant was collected to determine the POX and PPO activities.

POX activity, using guaiacol as substrate, was determined according to the method of Nagle and Haard $^{22}$. The supernatant $(0.05 \mathrm{ml})$ was mixed with $2.3 \mathrm{ml}$ of $0.01 \mathrm{M}$ sodium acetate buffer $(\mathrm{pH}$ 6.0), $0.05 \mathrm{ml}$ of $0.1 \%$ guaiacol $(\mathrm{V} / \mathrm{V})$ and $0.1 \mathrm{ml}$ of $0.1 \% \mathrm{H}_{2} \mathrm{O}_{2}(\mathrm{~V} / \mathrm{V})$. The mixture was kept at $25 \pm 1{ }^{\circ} \mathrm{C}$ for $5 \mathrm{~min}$ and the absorbance was measured at $470 \mathrm{~nm}$. One unit of enzyme activity was defined as the amount of enzyme that caused a change of 0.01 in absorbance per minute.

PPO activity, using catechol as substrate, was determined according to the method of Jiang and $\mathrm{Fu}^{23}$. The supernatant $(0.5 \mathrm{ml})$ was mixed with $1.3 \mathrm{ml}$ of $0.05 \mathrm{M} \mathrm{K}_{3} \mathrm{PO}_{4}$ buffer (pH 7.5) and $0.2 \mathrm{ml}$ of $0.2 \mathrm{M}$ catechol. The mixture was kept at $25 \pm 1{ }^{\circ} \mathrm{C}$ for $5 \mathrm{~min}$ and the absorbance was measured at $420 \mathrm{~nm}$. One unit of enzyme activity was defined as the amount of enzyme that caused a change of 0.01 in absorbance per minute.

Protein content was assayed based on the method of Lowry et $\mathrm{al}^{24}$ using BSA as a standard.

\section{Statistical analysis}

The experiments were arranged in a completely randomized design with three replications. Data were analysed by one-way ANOVA using statistical packages for the social science (SPSS) version 15.0. Tukey's Honestly Significant Differences (Tukey's HSD) were calculated to compare the significant effects at $p \leqslant 0.05$.

\section{RESULTS}

\section{Growth performances}

PBZ pretreatment significantly reduced the SH by $3-12 \%$, but increased root length (RL) by $7-24 \%$ of seedlings grown under a non-saline condition (Table 1). SFW and SDW of rice seedlings pretreated with PBZ under a non-saline condition sig- 
Table 1 Fresh weight (FW), dry weight (DW), shoot height (SH), and root length (RL) in PTT1 rice seedlings with or without pretreatment with $15 \mathrm{mg} / \mathrm{PBZ}$ and subsequently exposed to 0 or $150 \mathrm{mM} \mathrm{NaCl}$ for $-7,0,1,3,5,7,9$, and 12 days.

\begin{tabular}{|c|c|c|c|c|c|c|}
\hline Day & $\mathrm{PBZ}(\mathrm{mg} / 1)$ & $\mathrm{NaCl}(\mathrm{mM})$ & FW (mg) & DW (mg) & $\mathrm{SH}(\mathrm{cm})$ & $\mathrm{RL}(\mathrm{cm})$ \\
\hline-7 & 0 & 0 & $167.9 \pm 6.6$ & $19.9 \pm 0.56$ & $14.78 \pm 0.06$ & $9.92 \pm 0.07$ \\
\hline 0 & $\begin{array}{c}0 \\
15\end{array}$ & $\begin{array}{l}0 \\
0\end{array}$ & $\begin{array}{l}437.1 \pm 7.1^{\mathrm{a}} \\
443.6 \pm 5.2^{\mathrm{a}}\end{array}$ & $\begin{array}{l}52.6 \pm 0.43^{\mathrm{a}} \\
52.7 \pm 0.35^{\mathrm{a}}\end{array}$ & $\begin{array}{l}19.32 \pm 0.08^{\mathrm{a}} \\
18.20 \pm 0.07^{\mathrm{b}}\end{array}$ & $\begin{array}{l}11.94 \pm 0.06^{\mathrm{b}} \\
14.22 \pm 0.08^{\mathrm{a}}\end{array}$ \\
\hline 1 & $\begin{array}{c}0 \\
15 \\
0 \\
15\end{array}$ & $\begin{array}{c}0 \\
0 \\
150 \\
150\end{array}$ & $\begin{array}{l}463.3 \pm 9.4^{\mathrm{a}} \\
472.5 \pm 8.9^{\mathrm{a}} \\
454.5 \pm 9.6^{\mathrm{a}} \\
473.1 \pm 9.7^{\mathrm{a}}\end{array}$ & $\begin{array}{l}54.6 \pm 0.42^{\mathrm{a}} \\
56.5 \pm 0.61^{\mathrm{a}} \\
54.2 \pm 0.42^{\mathrm{a}} \\
56.1 \pm 0.51^{\mathrm{a}}\end{array}$ & $\begin{array}{l}19.84 \pm 0.07^{\mathrm{a}} \\
18.84 \pm 0.08^{\mathrm{b}} \\
19.60 \pm 0.05^{\mathrm{a}} \\
18.76 \pm 0.09^{\mathrm{b}}\end{array}$ & $\begin{array}{l}12.02 \pm 0.07^{\mathrm{b}} \\
14.92 \pm 0.08^{\mathrm{a}} \\
11.80 \pm 0.06^{\mathrm{b}} \\
14.84 \pm 0.07^{\mathrm{a}}\end{array}$ \\
\hline 3 & $\begin{array}{c}0 \\
15 \\
0 \\
15\end{array}$ & $\begin{array}{c}0 \\
0 \\
150 \\
150\end{array}$ & $\begin{array}{c}697 \pm 12^{\mathrm{a}} \\
700.4 \pm 5.7^{\mathrm{a}} \\
490 \pm 12^{\mathrm{b}} \\
684.8 \pm 9.8^{\mathrm{a}}\end{array}$ & $\begin{array}{l}82.9 \pm 0.99^{\mathrm{a}} \\
83.0 \pm 0.68^{\mathrm{a}} \\
58.9 \pm 0.68^{\mathrm{b}} \\
82.1 \pm 0.67^{\mathrm{a}}\end{array}$ & $\begin{array}{l}22.50 \pm 0.06^{\mathrm{a}} \\
21.92 \pm 0.05^{\mathrm{ab}} \\
20.44 \pm 0.08^{\mathrm{b}} \\
21.20 \pm 0.04^{\mathrm{ab}}\end{array}$ & $\begin{array}{l}14.10 \pm 0.06^{\mathrm{b}} \\
16.82 \pm 0.08^{\mathrm{a}} \\
11.70 \pm 0.09^{\mathrm{c}} \\
16.36 \pm 0.08^{\mathrm{a}}\end{array}$ \\
\hline 5 & $\begin{array}{c}0 \\
15 \\
0 \\
15\end{array}$ & $\begin{array}{c}0 \\
0 \\
150 \\
150\end{array}$ & $\begin{array}{c}1121 \pm 21^{\mathrm{a}} \\
1139 \pm 20^{\mathrm{a}} \\
626 \pm 12^{\mathrm{c}} \\
1047 \pm 26^{\mathrm{b}}\end{array}$ & $\begin{array}{l}133.8 \pm 1.3^{\mathrm{a}} \\
135.2 \pm 1.3^{\mathrm{a}} \\
75.7 \pm 0.71^{\mathrm{c}} \\
126.0 \pm 2.2^{\mathrm{b}}\end{array}$ & $\begin{array}{l}27.68 \pm 0.07^{\mathrm{a}} \\
25.72 \pm 0.06^{\mathrm{b}} \\
21.90 \pm 0.08^{\mathrm{c}} \\
24.22 \pm 0.09^{\mathrm{b}}\end{array}$ & $\begin{array}{l}18.24 \pm 0.07^{\mathrm{b}} \\
19.80 \pm 0.10^{\mathrm{a}} \\
11.48 \pm 0.09^{\mathrm{c}} \\
19.56 \pm 0.05^{\mathrm{a}}\end{array}$ \\
\hline 7 & $\begin{array}{c}0 \\
15 \\
0 \\
15\end{array}$ & $\begin{array}{c}0 \\
0 \\
150 \\
150\end{array}$ & $\begin{aligned} 1763 & \pm 18^{\mathrm{a}} \\
1776 & \pm 16^{\mathrm{a}} \\
839.2 & \pm 9.5^{\mathrm{c}} \\
1589 & \pm 24^{\mathrm{b}}\end{aligned}$ & $\begin{array}{c}210.9 \pm 1.7^{\mathrm{a}} \\
209.9 \pm 0.95^{\mathrm{a}} \\
102.2 \pm 1.2^{\mathrm{c}} \\
192.8 \pm 1.6^{\mathrm{b}}\end{array}$ & $\begin{array}{l}31.52 \pm 0.07^{\mathrm{a}} \\
27.66 \pm 0.06^{\mathrm{b}} \\
22.24 \pm 0.09^{\mathrm{c}} \\
25.42 \pm 0.07^{\mathrm{b}}\end{array}$ & $\begin{array}{l}21.24 \pm 0.08^{\mathrm{b}} \\
23.42 \pm 0.08^{\mathrm{a}} \\
11.58 \pm 0.07^{\mathrm{c}} \\
21.54 \pm 0.06^{\mathrm{b}}\end{array}$ \\
\hline 9 & $\begin{array}{c}0 \\
15 \\
0 \\
15\end{array}$ & $\begin{array}{c}0 \\
0 \\
150 \\
150\end{array}$ & $\begin{array}{c}2459 \pm 20^{\mathrm{a}} \\
2463 \pm 16^{\mathrm{a}} \\
840 \pm 16^{\mathrm{c}} \\
1779 \pm 15^{\mathrm{b}}\end{array}$ & $\begin{array}{l}293.1 \pm 1.7^{\mathrm{a}} \\
291.9 \pm 1.3^{\mathrm{a}} \\
103.1 \pm 1.7^{\mathrm{c}} \\
216.9 \pm 2.3^{\mathrm{b}}\end{array}$ & $\begin{array}{l}33.42 \pm 0.08^{\mathrm{a}} \\
30.24 \pm 0.06^{\mathrm{b}} \\
21.80 \pm 0.09^{\mathrm{d}} \\
26.24 \pm 0.07^{\mathrm{c}}\end{array}$ & $\begin{array}{l}23.40 \pm 0.10^{\mathrm{b}} \\
25.20 \pm 0.06^{\mathrm{a}} \\
11.57 \pm 0.07^{\mathrm{c}} \\
22.26 \pm 0.07^{\mathrm{b}}\end{array}$ \\
\hline 12 & $\begin{array}{c}0 \\
15 \\
0 \\
15\end{array}$ & $\begin{array}{c}0 \\
0 \\
150 \\
150\end{array}$ & $\begin{aligned} 3289 & \pm 16^{\mathrm{a}} \\
3309 & \pm 18^{\mathrm{a}} \\
831.2 & \pm 9.3^{\mathrm{c}} \\
1781 & \pm 15^{\mathrm{b}}\end{aligned}$ & $\begin{array}{l}386.1 \pm 1.8^{\mathrm{a}} \\
394.9 \pm 1.6^{\mathrm{a}} \\
103.6 \pm 1.2^{\mathrm{c}} \\
220.7 \pm 1.4^{\mathrm{b}}\end{array}$ & $\begin{array}{l}34.22 \pm 0.10^{\mathrm{a}} \\
32.12 \pm 0.08^{\mathrm{b}} \\
20.64 \pm 0.11^{\mathrm{d}} \\
27.58 \pm 0.09^{\mathrm{c}}\end{array}$ & $\begin{array}{l}24.96 \pm 0.07^{\mathrm{b}} \\
26.78 \pm 0.09^{\mathrm{a}} \\
11.52 \pm 0.08^{\mathrm{d}} \\
22.22 \pm 0.07^{\mathrm{c}}\end{array}$ \\
\hline
\end{tabular}

Values represent mean $\pm \mathrm{SE}(n=3)$. Different letters in each column show significant difference at $p \leqslant 0.05$, according to Tukey's HSD.

nificantly decreased by $5-10 \%$, whereas RFW and RDW significantly increased by $10-20 \%$ compared with non-PBZ treated seedlings (Table 2).

Fresh weight (FW), dry weight (DW), SH, RL, SFW, RFW, SDW, and RDW in rice seedlings were significantly reduced from day 3 after exposed to $150 \mathrm{mM} \mathrm{NaCl}$ as compared to those of the control (Tables 1 and 2). On day 7 after salt stress, FW, DW, SH, RL, SFW, RFW, SDW, and RDW of NaClstressed seedlings declined to $52,52,29,46,52$, 53,51 , and $53 \%$, respectively, as compared to the control, and these parameters decreased to 75,73 , $40,54,75,75,72$, and $75 \%$, respectively, on day 12 of salinization.

Pretreatment of seedlings with PBZ $(15 \mathrm{mg} / 1$ PBZ) significantly enhanced FW, DW, SH, RL, SFW, RFW, SDW, and RDW compared with controlled seedlings (water foliar spray) under salt stress (Ta- bles 1 and 2). FW, DW, SH, RL, SFW, RFW, SDW, and RDW of PBZ pretreated seedlings were $89,89,14$, $86,68,115,66$, and $128 \%$ higher than those of PBZ untreated seedlings on day 7 of salt stress, and 114, $113,34,93,89,146,84$, and $167 \%$, respectively, on day 12. The reduction in FW, DW, SL, RL, SFW, RFW, SDW and RDW in PBZ pretreated seedlings under $\mathrm{NaCl}$ stress on day 7 were only 10, 9, 19, 0, 20, 0 , 18 , and $0 \%$, respectively, as compared to the control under normal condition and on day 12 were 46, 43, $19,11,52,38,49$, and $33 \%$, respectively.

\section{Photosynthetic pigments}

Pretreatment with $15 \mathrm{mg} / \mathrm{l} \mathrm{PBZ}$ significantly enhanced $\mathrm{Chl}_{\mathrm{a}}, \mathrm{Ch}_{\mathrm{b}}, \mathrm{TC}$, and $\mathrm{C}_{\mathrm{x}+\mathrm{c}}$ contents of the seedlings grown under normal condition by $8-38 \%$ (Table 3). $\mathrm{Chl}_{\mathrm{a}}, \mathrm{Chl}_{\mathrm{b}}, \mathrm{TC}$, and $\mathrm{C}_{\mathrm{x}+\mathrm{c}}$ contents of seedlings were significantly decreased by salt stress 
Table 2 Shoot fresh weight (SFW), root fresh weight (RFW), shoot dry weight (SDW) and root dry weight (RDW) in PTT1 rice seedlings with or without pretreatment with $15 \mathrm{mg} / 1 \mathrm{PBZ}$ and subsequently exposed to 0 or $150 \mathrm{mM} \mathrm{NaCl}$ for $-7,0,1,3,5,7,9$, and 12 days.

\begin{tabular}{|c|c|c|c|c|c|c|}
\hline Day & PBZ (mg/l) & $\mathrm{NaCl}(\mathrm{mM})$ & SFW (mg) & RFW (mg) & SDW (mg) & $\mathrm{RDW}$ (mg) \\
\hline-7 & 0 & 0 & $90.9 \pm 2.6$ & $77.0 \pm 5.3$ & $12.3 \pm 0.12$ & $7.6 \pm 0.23$ \\
\hline 0 & $\begin{array}{c}0 \\
15\end{array}$ & $\begin{array}{l}0 \\
0\end{array}$ & $\begin{array}{l}236.7 \pm 2.7^{\mathrm{a}} \\
219.9 \pm 2.6^{\mathrm{b}}\end{array}$ & $\begin{array}{l}200.4 \pm 6.0^{\mathrm{b}} \\
223.7 \pm 5.3^{\mathrm{a}}\end{array}$ & $\begin{array}{l}32.3 \pm 0.89^{\mathrm{a}} \\
30.2 \pm 0.96^{\mathrm{a}}\end{array}$ & $\begin{array}{l}20.3 \pm 0.64^{\mathrm{a}} \\
22.6 \pm 0.86^{\mathrm{a}}\end{array}$ \\
\hline 1 & $\begin{array}{c}0 \\
15 \\
0 \\
15\end{array}$ & $\begin{array}{c}0 \\
0 \\
150 \\
150\end{array}$ & $\begin{array}{l}250.7 \pm 3.3^{\mathrm{a}} \\
233.2 \pm 2.3^{\mathrm{b}} \\
246.3 \pm 2.8^{\mathrm{a}} \\
233.4 \pm 3.0^{\mathrm{b}}\end{array}$ & $\begin{array}{l}212.6 \pm 6.8^{\mathrm{b}} \\
239.3 \pm 7.1^{\mathrm{a}} \\
208.2 \pm 8.2^{\mathrm{b}} \\
239.8 \pm 9.0^{\mathrm{a}}\end{array}$ & $\begin{array}{c}33.6 \pm 0.97^{\mathrm{a}} \\
31.8 \pm 0.55^{\mathrm{a}} \\
33.4 \pm 0.90^{\mathrm{a}} \\
31.7 \pm 1.3^{\mathrm{a}}\end{array}$ & $\begin{array}{l}21.0 \pm 0.75^{\mathrm{b}} \\
24.7 \pm 0.80^{\mathrm{a}} \\
20.7 \pm 0.75^{\mathrm{b}} \\
24.3 \pm 0.94^{\mathrm{a}}\end{array}$ \\
\hline 3 & $\begin{array}{c}0 \\
15 \\
0 \\
15\end{array}$ & $\begin{array}{c}0 \\
0 \\
150 \\
150\end{array}$ & $\begin{array}{l}377.0 \pm 3.2^{\mathrm{a}} \\
344.2 \pm 4.9^{\mathrm{b}} \\
265.0 \pm 4.0^{\mathrm{c}} \\
336.3 \pm 2.9^{\mathrm{b}}\end{array}$ & $\begin{array}{l}320.1 \pm 8.0^{\mathrm{b}} \\
356.1 \pm 4.2^{\mathrm{a}} \\
225.4 \pm 8.8^{\mathrm{c}} \\
348.5 \pm 7.0^{\mathrm{a}}\end{array}$ & $\begin{array}{c}51.0 \pm 1.5^{\mathrm{a}} \\
47.0 \pm 1.1^{\mathrm{ab}} \\
36.7 \pm 0.51^{\mathrm{c}} \\
45.8 \pm 0.78^{\mathrm{b}}\end{array}$ & $\begin{array}{c}31.9 \pm 0.55^{\mathrm{b}} \\
35.9 \pm 1.5^{\mathrm{a}} \\
22.3 \pm 0.82^{\mathrm{c}} \\
36.4 \pm 0.80^{\mathrm{a}}\end{array}$ \\
\hline 5 & $\begin{array}{c}0 \\
15 \\
0 \\
15\end{array}$ & $\begin{array}{c}0 \\
0 \\
150 \\
150\end{array}$ & $\begin{array}{l}602.7 \pm 4.7^{\mathrm{a}} \\
558.7 \pm 5.7^{\mathrm{b}} \\
343.4 \pm 4.4^{\mathrm{c}} \\
513.2 \pm 9.3^{\mathrm{b}}\end{array}$ & $\begin{array}{c}518.6 \pm 5.1^{\mathrm{b}} \\
580 \pm 11^{\mathrm{a}} \\
283 \pm 13^{\mathrm{c}} \\
534 \pm 12^{\mathrm{b}}\end{array}$ & $\begin{array}{c}81.9 \pm 1.7^{\mathrm{a}} \\
76.6 \pm 1.7^{\mathrm{a}} \\
47.6 \pm 0.97^{\mathrm{c}} \\
70.4 \pm 1.3^{\mathrm{b}}\end{array}$ & $\begin{array}{c}51.9 \pm 1.7^{\mathrm{b}} \\
58.7 \pm 1.2^{\mathrm{a}} \\
28.1 \pm 0.98^{\mathrm{c}} \\
55.6 \pm 1.7^{\mathrm{ab}}\end{array}$ \\
\hline 7 & $\begin{array}{c}0 \\
15 \\
0 \\
15\end{array}$ & $\begin{array}{c}0 \\
0 \\
150 \\
150\end{array}$ & $\begin{array}{l}963.8 \pm 4.2^{\mathrm{a}} \\
869.1 \pm 5.8^{\mathrm{b}} \\
461.2 \pm 5.6^{\mathrm{d}} \\
776.2 \pm 5.5^{\mathrm{c}}\end{array}$ & $\begin{array}{c}800 \pm 15^{\mathrm{b}} \\
906.8 \pm 9.8^{\mathrm{a}} \\
378.0 \pm 9.5^{\mathrm{c}} \\
812 \pm 12^{\mathrm{b}}\end{array}$ & $\begin{array}{c}131.3 \pm 2.3^{\mathrm{a}} \\
118.5 \pm 2.8^{\mathrm{b}} \\
64.8 \pm 1.9^{\mathrm{d}} \\
107.5 \pm 3.1^{\mathrm{c}}\end{array}$ & $\begin{array}{l}79.6 \pm 1.5^{\mathrm{b}} \\
91.5 \pm 1.3^{\mathrm{a}} \\
37.4 \pm 1.4^{\mathrm{c}} \\
85.3 \pm 1.6^{\mathrm{ab}}\end{array}$ \\
\hline 9 & $\begin{array}{c}0 \\
15 \\
0 \\
15\end{array}$ & $\begin{array}{c}0 \\
0 \\
150 \\
150\end{array}$ & $\begin{array}{c}1325.0 \pm 4.6^{\mathrm{a}} \\
1211.0 \pm 5.0^{\mathrm{b}} \\
464.8 \pm 5.5^{\mathrm{d}} \\
864.9 \pm 5.1^{\mathrm{c}}\end{array}$ & $\begin{array}{c}1134 \pm 12^{\mathrm{b}} \\
1252.4 \pm 9.6^{\mathrm{a}} \\
375.3 \pm 7.5^{\mathrm{d}} \\
914.0 \pm 7.6^{\mathrm{c}}\end{array}$ & $\begin{array}{c}179.6 \pm 3.4^{\mathrm{a}} \\
165.5 \pm 1.6^{\mathrm{b}} \\
66.8 \pm 1.3^{\mathrm{d}} \\
121.5 \pm 3.1^{\mathrm{c}}\end{array}$ & $\begin{array}{c}113.3 \pm 1.1^{\mathrm{b}} \\
126.4 \pm 0.94^{\mathrm{a}} \\
36.3 \pm 1.1^{\mathrm{d}} \\
95.5 \pm 2.2^{\mathrm{c}}\end{array}$ \\
\hline 12 & $\begin{array}{c}0 \\
15 \\
0 \\
15\end{array}$ & $\begin{array}{c}0 \\
0 \\
150 \\
150\end{array}$ & $\begin{array}{c}1801.5 \pm 5.1^{\mathrm{a}} \\
1621.9 \pm 6.4^{\mathrm{b}} \\
458.4 \pm 4.0^{\mathrm{d}} \\
864.2 \pm 7.1^{\mathrm{c}}\end{array}$ & $\begin{array}{c}1487.7 \pm 9.3^{\mathrm{b}} \\
1688 \pm 11^{\mathrm{a}} \\
373 \pm 11^{\mathrm{d}} \\
917 \pm 15^{\mathrm{c}}\end{array}$ & $\begin{array}{c}240.3 \pm 4.3^{\mathrm{a}} \\
220.4 \pm 3.3^{\mathrm{b}} \\
67.1 \pm 2.2^{\mathrm{d}} \\
123.3 \pm 3.2^{\mathrm{c}}\end{array}$ & $\begin{array}{c}145.8 \pm 2.0^{\mathrm{b}} \\
174.5 \pm 2.5^{\mathrm{a}} \\
36.5 \pm 1.4^{\mathrm{d}} \\
97.4 \pm 1.9^{\mathrm{c}}\end{array}$ \\
\hline
\end{tabular}

Values represent mean \pm SE $(n=3)$. Different letters in each column show significant difference at $p \leqslant 0.05$, according to Tukey's HSD.

during 5-12 days (Table 3). Contents of $\mathrm{Chl}_{\mathrm{a}}$, $\mathrm{Chl}_{\mathrm{b}}$, $\mathrm{TC}$, and $\mathrm{C}_{\mathrm{x}+\mathrm{c}}$ of NaCl-stressed seedlings decreased to $30,44,33$, and $32 \%$ on day 7 after salinization and declined by $47,56,49$, and $49 \%$, respectively, on day 12.

PBZ pretreatment alleviated the adverse effects on pigments and caused a significant prevention in $\mathrm{Chl}_{\mathrm{a}}, \mathrm{Chl}_{\mathrm{b}}, \mathrm{TC}$, and $\mathrm{C}_{\mathrm{x}+\mathrm{c}}$ contents in rice seedlings as compared to controlled seedlings (Table 3 ). The contents of $\mathrm{Chl}_{\mathrm{a}}, \mathrm{Chl}_{\mathrm{b}}, \mathrm{TC}$, and $\mathrm{C}_{\mathrm{x}+\mathrm{c}}$ of PBZ pretreated seedlings were $53,94,61$, and $43 \%$ higher than those of the controlled seedlings on day 7 after salt stress, and $49,104,60$, and $62 \%$, respectively, on day 12. The decreased $\mathrm{Chl}_{\mathrm{a}}, \mathrm{Chl}_{\mathrm{b}}, \mathrm{TC}$, and $\mathrm{C}_{\mathrm{x}+\mathrm{c}}$ contents in PBZ pretreated seedlings on day 7 after salt stress were only $0,0,0$, and $4 \%$ as compared with those in the control under normal condition and on day 12 they were reduced to $21,11,19$, and
$18 \%$, respectively.

\section{Total antioxidant capacities (TAC)}

Application of $15 \mathrm{mg} / 1 \mathrm{PBZ}$ to seedlings unexposed to salinity stress significantly increased TAC in both leaves and roots by 4-20\% (Figs. 1-3), as indicated by ABTS, DPPH, and FRAP assays. TAC in leaves and roots of rice seedlings significantly decreased after day 3 of salt stress (Figs. 1-3). On day 7 after salt stress, TAC in the leaves significantly decreased to 36,20 , and $37 \%$ for ABTS, DPPH, and FRAP assays, respectively; and these parameters declined to 54 , 44 , and $54 \%$, respectively, on day 12 . In roots, TAC decreased to 22,18 , and $25 \%$ by ABTS, DPPH, and FRAP assays, respectively, on day 7 , and they were 43,40 , and $38 \%$, respectively, on day 12 after salt stress (Figs. 1-3).

Pretreatment seedlings with PBZ significantly 
Table 3 Chlorophyll a $\left(\mathrm{Chl}_{\mathrm{a}}\right)$, chlorophyll b $\left(\mathrm{Chl}_{\mathrm{b}}\right)$, total chlorophyll (TC), and total carotenoid $\left(\mathrm{C}_{\mathrm{x}+\mathrm{c}}\right)$ contents in PTT1 rice seedlings with or without pretreatment with $15 \mathrm{mg} / 1 \mathrm{PBZ}$ and subsequently exposed to 0 or $150 \mathrm{mM} \mathrm{NaCl}$ for -7 , $0,1,3,5,7,9$, and 12 days.

\begin{tabular}{|c|c|c|c|c|c|c|}
\hline Day & PBZ (mg/l) & $\mathrm{NaCl}(\mathrm{mM})$ & $\mathrm{Chl}_{\mathrm{a}}(\mathrm{mg} / \mathrm{g} \mathrm{FW})$ & $\mathrm{Chl}_{\mathrm{b}}(\mathrm{mg} / \mathrm{g} \mathrm{FW})$ & TC (mg/g FW) & $\mathrm{C}_{\mathrm{x}+\mathrm{c}}(\mathrm{mg} / \mathrm{g} \mathrm{FW})$ \\
\hline-7 & 0 & 0 & $1.84 \pm 0.046$ & $0.54 \pm 0.033$ & $2.39 \pm 0.047$ & $0.49 \pm 0.034$ \\
\hline 0 & $\begin{array}{c}0 \\
15\end{array}$ & $\begin{array}{l}0 \\
0\end{array}$ & $\begin{array}{l}2.03 \pm 0.065^{\mathrm{b}} \\
2.29 \pm 0.061^{\mathrm{a}}\end{array}$ & $\begin{array}{l}0.63 \pm 0.032^{\mathrm{b}} \\
0.81 \pm 0.021^{\mathrm{a}}\end{array}$ & $\begin{array}{l}2.66 \pm 0.074^{\mathrm{b}} \\
3.11 \pm 0.080^{\mathrm{a}}\end{array}$ & $\begin{array}{l}0.58 \pm 0.032^{\mathrm{b}} \\
0.71 \pm 0.027^{\mathrm{a}}\end{array}$ \\
\hline 1 & $\begin{array}{c}0 \\
15 \\
0 \\
15\end{array}$ & $\begin{array}{c}0 \\
0 \\
150 \\
150\end{array}$ & $\begin{array}{l}2.10 \pm 0.156^{\mathrm{b}} \\
2.33 \pm 0.157^{\mathrm{a}} \\
2.04 \pm 0.150^{\mathrm{b}} \\
2.35 \pm 0.078^{\mathrm{a}}\end{array}$ & $\begin{array}{l}0.64 \pm 0.021^{b} \\
0.84 \pm 0.038^{a} \\
0.59 \pm 0.028^{b} \\
0.81 \pm 0.038^{a}\end{array}$ & $\begin{array}{l}2.75 \pm 0.064^{\mathrm{b}} \\
3.16 \pm 0.091^{\mathrm{a}} \\
2.63 \pm 0.053^{\mathrm{b}} \\
3.15 \pm 0.061^{\mathrm{a}}\end{array}$ & $\begin{array}{l}0.56 \pm 0.088^{\mathrm{b}} \\
0.72 \pm 0.030^{\mathrm{a}} \\
0.54 \pm 0.021^{\mathrm{b}} \\
0.70 \pm 0.035^{\mathrm{a}}\end{array}$ \\
\hline 3 & $\begin{array}{c}0 \\
15 \\
0 \\
15\end{array}$ & $\begin{array}{c}0 \\
0 \\
150 \\
150\end{array}$ & $\begin{array}{l}2.08 \pm 0.054^{\mathrm{ab}} \\
2.25 \pm 0.054^{\mathrm{a}} \\
1.86 \pm 0.074^{\mathrm{b}} \\
2.31 \pm 0.050^{\mathrm{a}}\end{array}$ & $\begin{array}{l}0.60 \pm 0.022^{\mathrm{b}} \\
0.83 \pm 0.033^{\mathrm{a}} \\
0.51 \pm 0.030^{\mathrm{b}} \\
0.85 \pm 0.027^{\mathrm{a}}\end{array}$ & $\begin{array}{l}2.68 \pm 0.042^{\mathrm{b}} \\
3.08 \pm 0.050^{\mathrm{a}} \\
2.37 \pm 0.096^{\mathrm{c}} \\
3.16 \pm 0.064^{\mathrm{a}}\end{array}$ & $\begin{array}{l}0.61 \pm 0.022^{a b} \\
0.70 \pm 0.037^{a} \\
0.50 \pm 0.034^{b} \\
0.67 \pm 0.024^{a}\end{array}$ \\
\hline 5 & $\begin{array}{c}0 \\
15 \\
0 \\
15\end{array}$ & $\begin{array}{c}0 \\
0 \\
150 \\
150\end{array}$ & $\begin{array}{l}2.03 \pm 0.094^{\mathrm{b}} \\
2.32 \pm 0.070^{\mathrm{a}} \\
1.59 \pm 0.077^{\mathrm{c}} \\
2.36 \pm 0.049^{\mathrm{a}}\end{array}$ & $\begin{array}{l}0.62 \pm 0.022^{\mathrm{b}} \\
0.80 \pm 0.039^{\mathrm{a}} \\
0.41 \pm 0.030^{\mathrm{c}} \\
0.81 \pm 0.021^{\mathrm{a}}\end{array}$ & $\begin{array}{l}2.64 \pm 0.109^{\mathrm{b}} \\
3.12 \pm 0.093^{\mathrm{a}} \\
1.99 \pm 0.101^{\mathrm{c}} \\
3.18 \pm 0.046^{\mathrm{a}}\end{array}$ & $\begin{array}{l}0.57 \pm 0.014^{\mathrm{bc}} \\
0.69 \pm 0.034^{\mathrm{a}} \\
0.46 \pm 0.019^{\mathrm{c}} \\
0.65 \pm 0.038^{\mathrm{ab}}\end{array}$ \\
\hline 7 & $\begin{array}{c}0 \\
15 \\
0 \\
15\end{array}$ & $\begin{array}{c}0 \\
0 \\
150 \\
150\end{array}$ & $\begin{array}{l}2.00 \pm 0.081^{\mathrm{b}} \\
2.28 \pm 0.075^{\mathrm{a}} \\
1.41 \pm 0.060^{\mathrm{c}} \\
2.15 \pm 0.042^{\mathrm{ab}}\end{array}$ & $\begin{array}{l}0.66 \pm 0.023^{\mathrm{b}} \\
0.78 \pm 0.021^{\mathrm{a}} \\
0.37 \pm 0.026^{\mathrm{c}} \\
0.72 \pm 0.036^{\mathrm{ab}}\end{array}$ & $\begin{array}{l}2.65 \pm 0.101^{\mathrm{b}} \\
3.05 \pm 0.071^{\mathrm{a}} \\
1.79 \pm 0.079^{\mathrm{c}} \\
2.87 \pm 0.056^{\mathrm{ab}}\end{array}$ & $\begin{array}{l}0.61 \pm 0.031^{\mathrm{b}} \\
0.68 \pm 0.025^{\mathrm{a}} \\
0.41 \pm 0.015^{\mathrm{c}} \\
0.59 \pm 0.043^{\mathrm{b}}\end{array}$ \\
\hline 9 & $\begin{array}{c}0 \\
15 \\
0 \\
15\end{array}$ & $\begin{array}{c}0 \\
0 \\
150 \\
150\end{array}$ & $\begin{array}{l}2.09 \pm 0.079^{\mathrm{ab}} \\
2.26 \pm 0.070^{\mathrm{a}} \\
1.27 \pm 0.053^{\mathrm{c}} \\
1.86 \pm 0.074^{\mathrm{b}}\end{array}$ & $\begin{array}{l}0.64 \pm 0.021^{\mathrm{b}} \\
0.84 \pm 0.024^{\mathrm{a}} \\
0.34 \pm 0.043^{\mathrm{c}} \\
0.62 \pm 0.021^{\mathrm{b}}\end{array}$ & $\begin{array}{l}2.73 \pm 0.077^{\mathrm{b}} \\
3.11 \pm 0.081^{\mathrm{a}} \\
1.61 \pm 0.071^{\mathrm{c}} \\
2.48 \pm 0.078^{\mathrm{b}}\end{array}$ & $\begin{array}{l}0.60 \pm 0.020^{\mathrm{b}} \\
0.73 \pm 0.023^{\mathrm{a}} \\
0.38 \pm 0.046^{\mathrm{c}} \\
0.54 \pm 0.017^{\mathrm{b}}\end{array}$ \\
\hline 12 & $\begin{array}{c}0 \\
15 \\
0 \\
15\end{array}$ & $\begin{array}{c}0 \\
0 \\
150 \\
150\end{array}$ & $\begin{array}{l}2.12 \pm 0.093^{\mathrm{b}} \\
2.27 \pm 0.059^{\mathrm{a}} \\
1.12 \pm 0.061^{\mathrm{d}} \\
1.67 \pm 0.078^{\mathrm{c}}\end{array}$ & $\begin{array}{l}0.64 \pm 0.026^{\mathrm{b}} \\
0.83 \pm 0.017^{\mathrm{a}} \\
0.28 \pm 0.034^{\mathrm{c}} \\
0.57 \pm 0.013^{\mathrm{b}}\end{array}$ & $\begin{array}{l}2.77 \pm 0.081^{\mathrm{b}} \\
3.10 \pm 0.051^{\mathrm{a}} \\
1.40 \pm 0.063^{\mathrm{d}} \\
2.24 \pm 0.085^{\mathrm{c}}\end{array}$ & $\begin{array}{l}0.63 \pm 0.025^{\mathrm{ab}} \\
0.66 \pm 0.048^{\mathrm{a}} \\
0.32 \pm 0.031^{\mathrm{c}} \\
0.52 \pm 0.026^{\mathrm{b}}\end{array}$ \\
\hline
\end{tabular}

Values represent mean $\pm \mathrm{SE}(n=3)$.Different letters in each column show significant difference at $p \leqslant 0.05$, according to Tukey's HSD.

improved TAC throughout salinity condition as compared to the untreated seedlings (Figs. 1-3). TAC in the leaves of PBZ treated seedlings were higher than those in the untreated seedlings by 46,20 , and $35 \%$, respectively, after 7 days of salt stress as analysed by ABTS, DPPH, and FRAP assays and they were 37,52 , and $50 \%$ higher than those of the control on day 12. TAC in the roots of seedlings treated with PBZ were 29, 21, and $27 \%$ higher than those of PBZ untreated roots on day 7 , and they were 35,53 , and 37\% higher by ABTS, DPPH, and FRAP assays, respectively, on day 12 after salt stress.

\section{Antioxidant activities}

PBZ pretreatment significantly enhanced antioxidant activities of POX and PPO by $7-35 \%$ in rice seedlings grown under a normal condition (Figs. 4, 5). The activities of POX and PPO in leaves and roots of PBZ untreated seedlings slightly increased during the first 3 days of salt stress and significantly decreased during a long period of salt exposure (Figs. 4, 5). Salt stress reduced activities of POX and PPO in the leaves of PBZ untreated seedlings by 10 and $9 \%$ on day 7 , and they were decreased by 16 and 15\% of the control on day 12 after exposure to salt stress. POX and PPO activities in the roots decreased by 6 and $8 \%$ of the control on day 7 and 11 and 16\% of the control on day 12 after salinization.

PBZ pretreatment significantly increased POX and PPO activities in comparison with PBZ untreated seedlings under saline condition. These activities greatly increased during the first 5 days after salinity and slightly decreased thereafter (Figs. 4, 5). POX and PPO activities in the leaves of PBZ treated seedling were higher than those in the untreated 

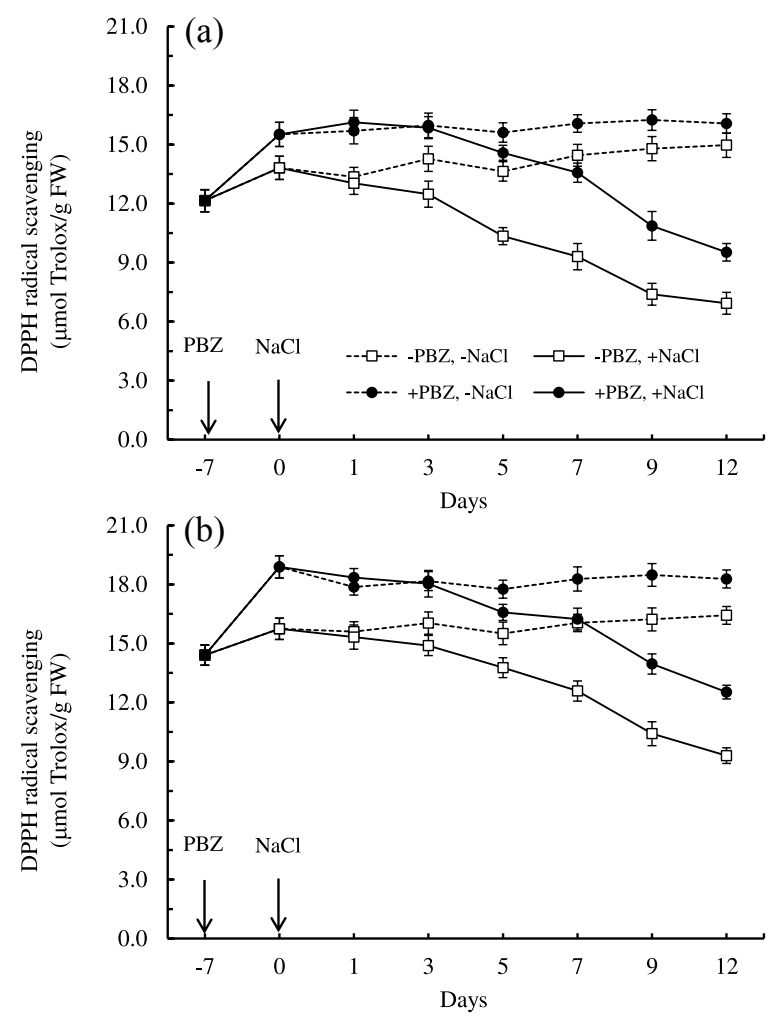

Fig. 1 Antioxidant capacity using DPPH assay in (a) leaves and (b) roots of PTT1 rice seedlings with or without pretreatment with $15 \mathrm{mg} / \mathrm{PBZ}$ and subsequently exposed to 0 or $150 \mathrm{mM} \mathrm{NaCl}$ for $-7,0,1,3,5,7,9$, and 12 days. Values represent mean $\pm \operatorname{SE}(n=3)$.

seedlings by 62 and $29 \%$ on day 7, and 24 and $19 \%$ on day 12 after salt stress (Figs. 4, 5). In the roots, POX and PPO activities of PBZ treated seedling were higher than PBZ untreated seedlings by 45 and $24 \%$ on day 7 , and 23 and $21 \%$ on day 12 after salt stress, respectively (Figs. 4, 5).

\section{DISCUSSION}

Salinity affects growth and development in several crop seedlings leading to a loss in productivity $3,25,26$. Severity of plant growth caused by salinity stress is indicated by the decrease in FW, DW, $\mathrm{SH}$, and RL. In the present study, $\mathrm{NaCl}$ stress significantly reduced FW, DW, SH, RL, SFW, RFW, SDW, and RDW of rice seedlings cv. PTT1 (Tables 1, 2), indicating that PTT1 is sensitive to salinity, and the results are compatible with Cha-um et $\mathrm{al}^{16}$ and Boriboonkaset et $\mathrm{al}^{26}$. Salt stress also significantly reduces growth of many other crop seedlings such as maize ${ }^{25}$, barley ${ }^{27}$, and wheat ${ }^{28}$.

PBZ application has been reported to mitigate
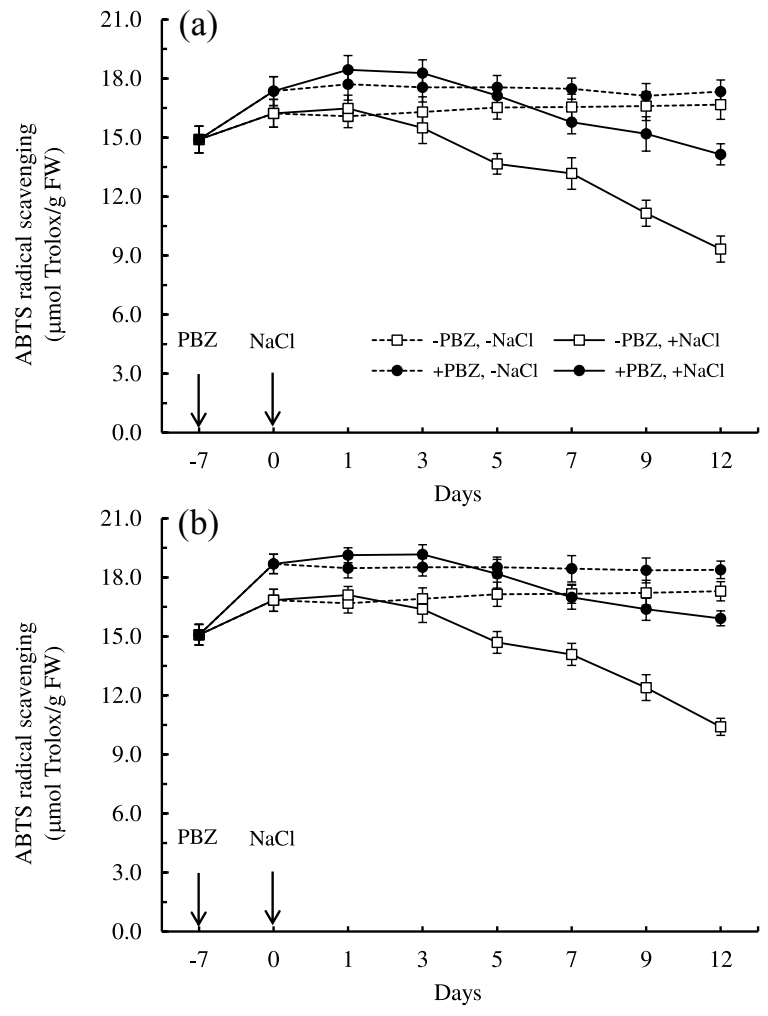

Fig. 2 Antioxidant capacity using ABTS assay in (a) leaves and (b) roots of PTT1 rice seedlings with or without pretreatment with $15 \mathrm{mg} / \mathrm{PBZ}$ and subsequently exposed to 0 or $150 \mathrm{mM} \mathrm{NaCl}$ for $-7,0,1,3,5,7,9$, and 12 days. Values represent mean $\pm \mathrm{SE}(n=3)$.

the adverse effects of salinity in many plants ${ }^{12,27,29}$. In this study, an application of PBZ by foliar spraying to rice seedlings cv. PTT1 significantly increased growth parameters, i.e., FW, DW, SH, RL, SFW, RFW, SDW, and RDW with an average of $82 \%$ as compared with those of seedlings without PBZ on day 7 of salt stress (Tables 1,2 ). It is possible that PBZ could reduce the adverse effect of salinity as previously reported in cowpea ${ }^{13}$, barley ${ }^{27}$, and wheat ${ }^{29}$. PBZ treatment promoted the growth of seedlings, especially root growth (Tables 1,2 ). It has been reported that better root growth and vigour stimulated by PBZ are important for plant adaptation under stress in wheat ${ }^{29}$. Increasing root growth by PBZ is also associated with an increased level of endogenous cytokinin that promotes plant growth and development and delays senescence in plants ${ }^{11}$. Stimulation of root growth against stress by PBZ has been reported in cowpea ${ }^{13}$ and barley ${ }^{27}$ for salt stress.

Photosynthesis is considered to be a primary 

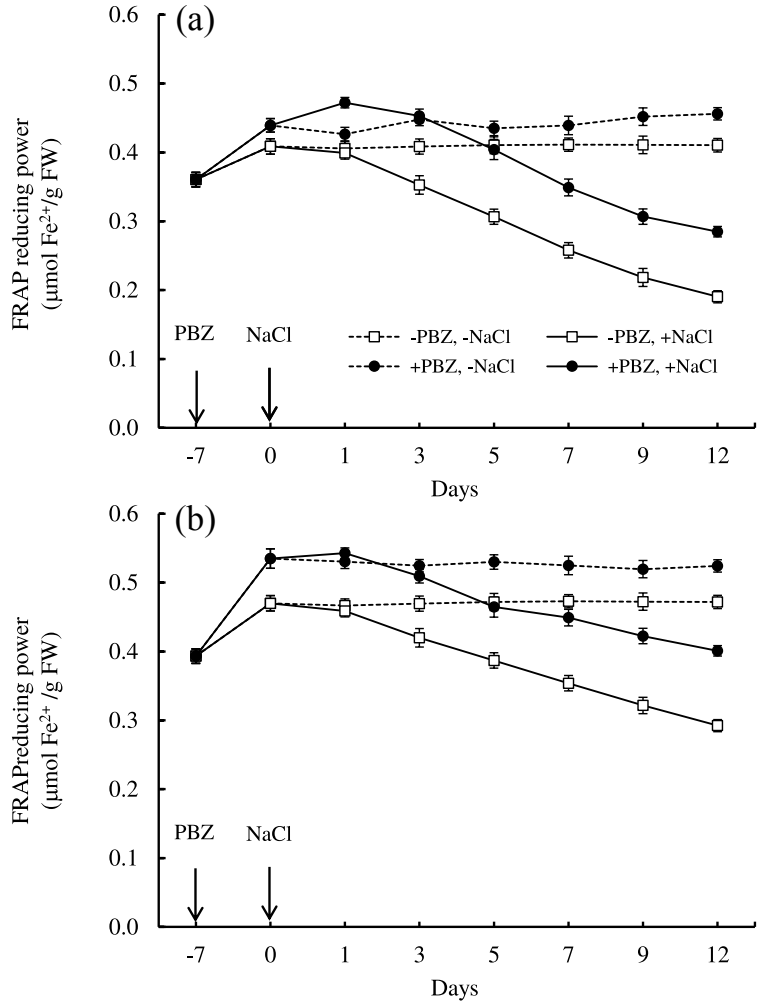

Fig. 3 Antioxidant capacity using FRAP assay in (a) leaves and (b) roots of PTT1 rice seedlings with or without pretreatment with $15 \mathrm{mg} / \mathrm{PBZ}$ and subsequently exposed to 0 or $150 \mathrm{mM} \mathrm{NaCl}$ for $-7,0,1,3,5,7,9$, and 12 days. Values represent mean $\pm \operatorname{SE}(n=3)$.

process affected by salinity, and plant growth is dependent on its efficiency ${ }^{3,4}$. In the present study, salt stress significantly reduced contents of $\mathrm{Chl}_{\mathrm{a}}$, $\mathrm{Chl}_{\mathrm{b}}$, TC, and $\mathrm{C}_{\mathrm{x}+\mathrm{c}}$ with increasing salt exposure time (Table 3). The result agrees with Chutipaijit et $\mathrm{al}^{5}$ and Roychoudhury et $\mathrm{al}^{10}$ who found that salt stress induced degradation of photosynthetic pigments. It has been reported that disruption of chloroplastic and thylakoid membranes and photosynthetic pigment degradation are stimulated by free radicals generated under salt stress. Thus decrease in photosynthetic pigments indicates a direct reduction in photosynthetic efficiency and hence reduces carbon fixation ${ }^{3}$. Moreover, chlorophyll and carotenoid concentrations have been used as sensitive indicators of the cellular metabolic state and physiological response of salt sensitive cultivars ${ }^{3}$. In this study, a decrease in photosynthetic pigments is highly correlated with seedling growth reduction ( $r=0.894-0.962)$ under salinity (Table 4), indicating that under salinity, photosynthesis of rice
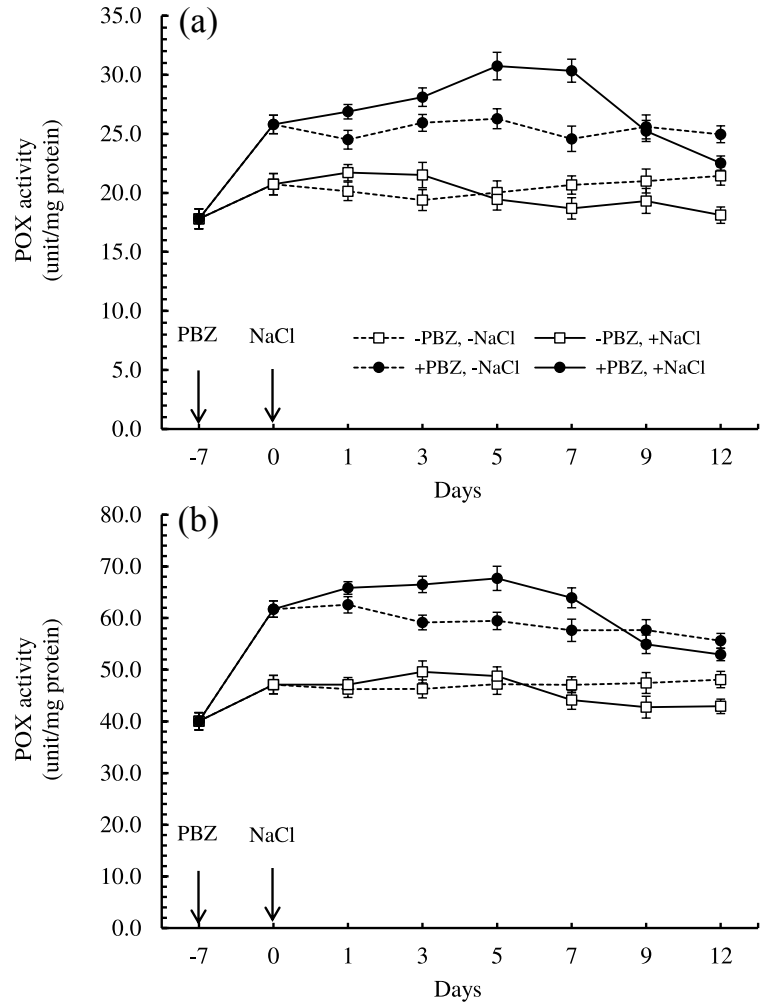

Fig. 4 POX activity in (a) leaves and (b) roots of PTT1 rice seedlings with or without pretreatment with $15 \mathrm{mg} / \mathrm{PBZ}$ and subsequently exposed to 0 or $150 \mathrm{mM} \mathrm{NaCl}$ for -7 , $0,1,3,5,7,9$, and 12 days. Values represent mean $\pm \mathrm{SE}$ $(n=3)$.

seedlings cv. PTT1 is interrupted. The parallel decreases on photosynthetic pigments and growth capacity of plants due to salt stress have been reported in maize ${ }^{3}$, sugarcane ${ }^{4}$, and wheat ${ }^{28}$.

In this experiment, PBZ pretreatment significantly enhanced and restored the photosynthetic pigment level in PTT1 rice seedlings exposed to salt stress. PBZ significantly enhanced photosynthetic pigment with the average of $63 \%$ as compared with the control on day 7 after salt stress (Table 3 ). It is possible that the increase in photosynthetic pigments in leaves stimulated by PBZ led to higher photosynthesis and higher growth ability of PTT1 rice seedlings under salt stress compared with those seedlings without PBZ treatment. The increase in chlorophyll and carotenoid contents by PBZ may be partly due to an increase in root growth (Tables 1,2), which is the major site of cytokinin biosynthesis ${ }^{11}$. It has been reported that high levels of cytokinins may stimulate chlorophyll biosynthesis for more efficient photosynthetic performance in 

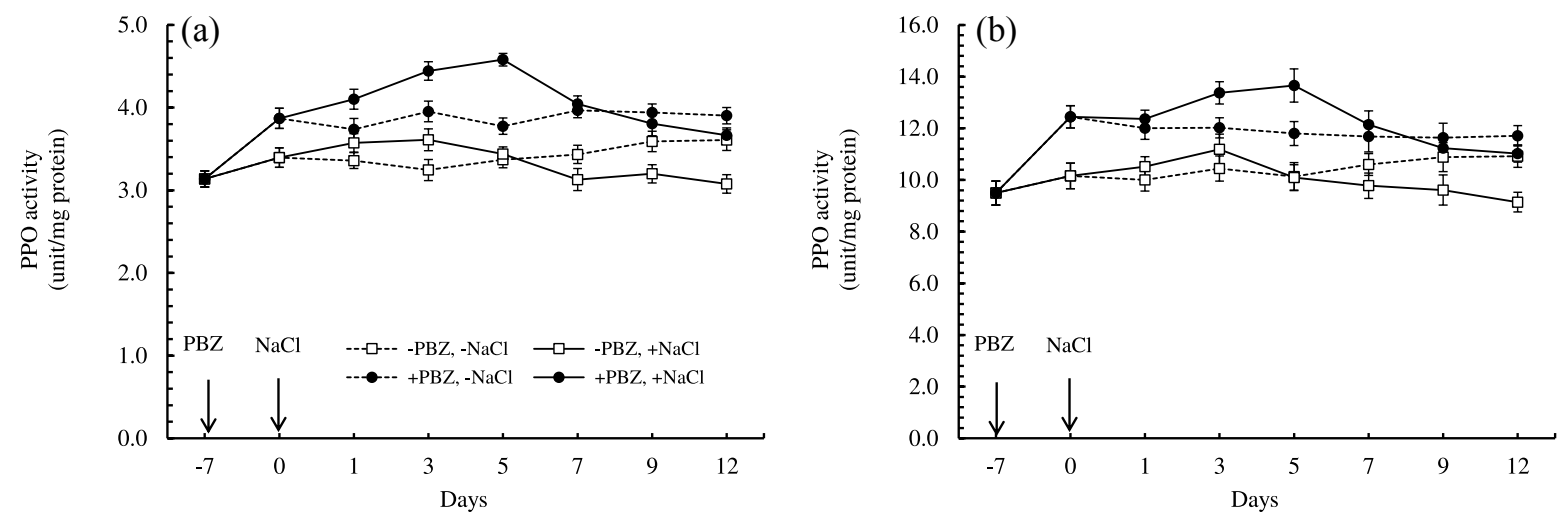

Fig. 5 PPO activity in (a) leaves and (b) roots of PTT1 rice seedlings with or without pretreatment with $15 \mathrm{mg} / 1 \mathrm{PBZ}$ and subsequently exposed to 0 or $150 \mathrm{mM} \mathrm{NaCl}$ for $-7,0,1,3,5,7,9$, and 12 days. Values represent mean $\pm \mathrm{SE}$ $(n=3)$.

plant defence against stress ${ }^{11}$. Enhancement of photosynthetic pigment in plants by PBZ has also been reported in barley ${ }^{27}$ and wheat ${ }^{28}$.

Salinity stress induces the production of ROS such as $\mathrm{O}_{2}^{--}, \mathrm{H}_{2} \mathrm{O}_{2}$, and $\mathrm{HO}^{\cdot}$. In the absence of a protective mechanism in plants, ROS cause serious damage to cell structure and function such as initiating lipid peroxidation and damaging DNA, proteins, and small molecules, especially in the chloroplasts and mitochondria ${ }^{6}$. Thus plants are equipped with enzymatic and non-enzymatic antioxidant systems to alleviate cellular damage caused by ROS $^{7}$. The total antioxidant efficiencies are represented by a series of antioxidant molecules that the plant uses against ROS formation. DPPH, ABTS, and FRAP assays were usually used to provide information on the antioxidant efficiencies of abiotic stressedseedlings. In the present study, antioxidant efficiencies determined by ABTS, DPPH, and FRAP assays in leaves and roots of PTT1 rice seedlings under salt stress decreased steadily throughout storage (Figs. 1-3), indicating that salt stress may cause a decrease in both enzymatic and non-enzymatic antioxidant activities for ROS scavenging. The con-

Table 4 Pearson correlation coefficients of growth performance, photosynthetic pigment and antioxidant efficiency in PTT1 rice seedlings exposed to $150 \mathrm{mM} \mathrm{NaCl}$ for 12 days.

\begin{tabular}{|c|c|c|c|c|c|c|c|c|c|c|c|c|c|c|c|c|c|c|c|c|c|c|}
\hline \multirow[t]{2}{*}{ Trait } & \multicolumn{22}{|c|}{$r$ value } \\
\hline & FW & DW & SL & $\mathrm{RL}$ & SFW & RFW & SDW & RDW & $\mathrm{Chl}_{\mathrm{a}}$ & $\mathrm{Chl}_{\mathrm{b}}$ & TC & $C_{x+c}$ & $\begin{array}{c}\text { DPPH } \\
-\mathrm{L}\end{array}$ & $\begin{array}{c}\text { DPPH } \\
-\mathrm{R}\end{array}$ & $\begin{array}{c}\mathrm{ABTS} \\
-\mathrm{L}\end{array}$ & $\begin{array}{c}\text { ABTS } \\
-\mathrm{R}\end{array}$ & $\begin{array}{l}\text { FRAP } \\
-\mathrm{L}\end{array}$ & $\begin{array}{c}\text { FRAP } \\
-\mathrm{R}\end{array}$ & $\begin{array}{c}\text { POX } \\
-\mathrm{L}\end{array}$ & $\begin{array}{c}\mathrm{POX} \\
-\mathrm{R}\end{array}$ & $\begin{array}{c}\text { PPO } \\
-\mathrm{L}\end{array}$ & $\begin{array}{c}\text { PPO } \\
-R\end{array}$ \\
\hline FW & 1 & & & & & & & & & & & & & & & & & & & & & \\
\hline DW & $0.999^{*}$ & 1 & & & & & & & & & & & & & & & & & & & & \\
\hline SL & $0.968^{*}$ & $0.968^{*}$ & 1 & & & & & & & & & & & & & & & & & & & \\
\hline $\mathrm{RL}$ & $0.956^{*}$ & $0.960^{*}$ & $0.981^{*}$ & 1 & & & & & & & & & & & & & & & & & & \\
\hline SFW & $0.998^{*}$ & $0.999^{*}$ & $0.966^{*}$ & $0.952^{*}$ & 1 & & & & & & & & & & & & & & & & & \\
\hline RFW & $0.999^{*}$ & $0.999^{*}$ & $0.969^{*}$ & $0.960^{*}$ & $0.999^{*}$ & 1 & & & & & & & & & & & & & & & & \\
\hline SDW & $0.997^{*}$ & $0.998^{*}$ & $0.969^{*}$ & $0.956^{*}$ & $0.999^{*}$ & $0.998^{*}$ & 1 & & & & & & & & & & & & & & & \\
\hline RDW & $0.998^{*}$ & $0.999^{*}$ & $0.966^{*}$ & $0.964^{*}$ & $0.997^{*}$ & $0.998^{*}$ & $0.997^{*}$ & 1 & & & & & & & & & & & & & & \\
\hline $\mathrm{Chl}_{\mathrm{a}}$ & $0.961^{*}$ & $0.962^{*}$ & $0.939 *$ & $0.949^{*}$ & $0.959^{*}$ & $0.962^{*}$ & $0.958^{*}$ & $0.965^{*}$ & 1 & & & & & & & & & & & & & \\
\hline $\mathrm{Chl}_{\mathrm{b}}^{\mathrm{d}}$ & $0.898^{*}$ & $0.904^{*}$ & $0.876^{*}$ & $0.917^{*}$ & $0.894^{*}$ & 0.901 " & $0.892^{*}$ & $0.911^{*}$ & 0.970 " & 1 & & & & & & & & & & & & \\
\hline TC & $0.946^{*}$ & $0.946^{*}$ & $0.925^{*}$ & $0.945^{*}$ & $0.944^{*}$ & $0.948^{*}$ & $0.943^{*}$ & $0.954^{*}$ & $0.997^{*}$ & $0.986^{*}$ & 1 & & & & & & & & & & & \\
\hline$C_{x+c}$ & $0.921^{*}$ & $0.919^{*}$ & $0.912^{*}$ & $0.921^{*}$ & $0.919^{*}$ & $0.922^{*}$ & $0.916^{*}$ & $0.922^{*}$ & $0.974^{*}$ & $0.980^{*}$ & $0.982^{*}$ & 1 & & & & & & & & & & \\
\hline DPPH-L & $0.960^{*}$ & $0.956^{*}$ & $0.925^{*}$ & $0.905^{*}$ & $0.961^{*}$ & $0.959^{*}$ & $0.956^{*}$ & $0.955^{*}$ & $0.975^{*}$ & $0.929^{*}$ & $0.967^{*}$ & $0.956^{*}$ & 1 & & & & & & & & & \\
\hline DPPH-R & $0.942^{*}$ & $0.938^{*}$ & $0.913^{*}$ & $0.883^{*}$ & $0.943^{*}$ & 0.941 & $0.939^{*}$ & $0.936^{*}$ & $0.963^{*}$ & $0.922^{*}$ & 0.956 & $0.965^{*}$ & $0.987^{*}$ & 1 & & & & & & & & \\
\hline ABTS-L & $0.953^{*}$ & $0.952^{*}$ & $0.952^{*}$ & $0.934^{*}$ & $0.952^{*}$ & 0.954 & $0.951^{*}$ & $0.952^{*}$ & 0.970 & $0.927^{*}$ & $0.963^{*}$ & $0.955^{*}$ & $0.965^{*}$ & 0.964 & 1 & & & & & & & \\
\hline ABTS-R & $0.933^{*}$ & $0.933^{*}$ & $0.946^{*}$ & $0.937^{*}$ & $0.931^{*}$ & $0.935^{*}$ & $0.931^{*}$ & $0.936^{*}$ & 0.970 & $0.945^{*}$ & $0.969^{*}$ & $0.969^{*}$ & $0.950^{*}$ & $0.958^{*}$ & $0.993^{*}$ & 1 & & & & & & \\
\hline FRAP-L & $0.961^{*}$ & $0.954^{*}$ & $0.943^{*}$ & $0.908^{*}$ & $0.962^{*}$ & $0.959^{*}$ & $0.957^{*}$ & $0.949^{*}$ & $0.953^{*}$ & $0.897^{*}$ & 0.941 & $0.941^{*}$ & $0.984^{*}$ & $0.968^{*}$ & $0.969^{*}$ & $0.948^{*}$ & 1 & & & & & \\
\hline FRAP-R & $0.952^{*}$ & $0.947^{*}$ & $0.945^{*}$ & $0.926^{*}$ & $0.951^{*}$ & $0.952^{*}$ & $0.949^{*}$ & $0.944^{*}$ & $0.960^{*}$ & $0.936^{*}$ & $0.959^{*}$ & $0.979^{*}$ & $0.972^{*}$ & $0.978^{*}$ & $0.969^{*}$ & $0.967^{*}$ & $0.978^{*}$ & 1 & & & & \\
\hline POX-L & $0.693^{*}$ & $0.705^{*}$ & $0.644^{\dagger}$ & $0.729^{*}$ & $0.689^{*}$ & $0.698^{*}$ & $0.690^{*}$ & $0.724^{*}$ & $0.837^{*}$ & $0.888^{*}$ & $0.860^{*}$ & $0.810^{*}$ & $0.744^{*}$ & $0.737^{*}$ & $0.731^{*}$ & $0.766^{*}$ & $0.654^{\dagger}$ & $0.706^{*}$ & 1 & & & \\
\hline POX-R & $0.696^{*}$ & $0.702^{*}$ & $0.660^{\dagger}$ & $0.718^{*}$ & $0.693^{*}$ & 0.700 & $0.689^{*}$ & $0.717^{*}$ & $0.856^{*}$ & $0.916^{*}$ & $0.881^{*}$ & $0.871^{*}$ & $0.802^{*}$ & $0.806^{*}$ & $0.778^{*}$ & $0.812^{*}$ & $0.729^{*}$ & $0.781^{*}$ & $0.956^{*}$ & 1 & & \\
\hline PPO-L & $0.711^{*}$ & $0.719^{*}$ & $0.688^{*}$ & $0.753^{*}$ & $0.707^{*}$ & $0.714^{*}$ & $0.705^{*}$ & $0.737^{*}$ & $0.865^{*}$ & $0.904^{*}$ & $0.884^{*}$ & $0.840^{*}$ & $0.789^{*}$ & $0.772^{*}$ & $0.797^{*}$ & $0.824^{*}$ & $0.735^{*}$ & $0.749^{*}$ & $0.946^{*}$ & $0.957^{*}$ & 1 & \\
\hline PPO-R & $0.725^{*}$ & $0.731^{*}$ & $0.705^{*}$ & $0.757^{*}$ & $0.721^{*}$ & $0.728^{*}$ & $0.718^{*}$ & $0.746^{*}$ & $0.881^{*}$ & $0.924^{*}$ & 0.901 & $0.877^{*}$ & $0.824^{*}$ & 0.822 & $0.820^{*}$ & $0.852^{*}$ & $0.762^{*}$ & $0.789^{*}$ & $0.936^{*}$ & $0.966^{*}$ & $0.980^{*}$ & 1 \\
\hline
\end{tabular}

$\dagger$ Significant at $p<0.05$ probability level.

" Significant at $p<0.01$ probability level. 
tinuous decrease in ABTS and DPPH radicals scavenging indicated that the ROS scavenging capacity in plants was deteriorated and the decrease in FRAP also indicated that electron donation capacity or reducing power in plant lost efficacy ${ }^{30}$. The results of this study are consistent with those of Sharma et $\mathrm{al}^{8}$ and Li et $\mathrm{al}^{31}$ who reported that antioxidant efficiency declined during salt stress in rice and wheat, respectively.

The decline in antioxidant efficiencies (Figs. 13) correlated with decreased photosynthetic pigments ( $r=0.897-0.979)$ and growth reduction $(r=0.931-0.962)$ of rice seedlings under salt stress (Table 4). It is possible that salt stress reduced antioxidant efficiency in seedlings by inducing ROS accumulation leading to the degradation of photosynthetic pigments and growth reduction. In this experiment, the antioxidant capacity in the roots was higher than that in the leaves under salinity stress (Figs. 1-3) indicating that the ability to scavenge ROS in the roots was better than that in the leaves.

PBZ pretreatment greatly increased and restored antioxidant efficiencies in leaves and roots of PTT1 rice seedlings under salinity (Figs. 1-3). The increased TAC by PBZ possibly reduces ROSinduced damage to photosynthetic system, leading to the growth ability under salt stress (Figs. 13 and Tables 1-3). In comparison, increasing of antioxidant capacity by PBZ in the roots was higher than that in the leaves because PBZ might stimulate the increase in root growth, which is the site of antioxidant biosynthesis. The higher antioxidant in the roots than that in the leaves has been observed in rice cultivars Malviya-36 and Pant- $12^{32}$, and Kentucky bluegrass ${ }^{33}$. However, the underlying mechanism of PBZ on enhancing antioxidant defence system has not been clearly understood ${ }^{14}$. It is possible that PBZ might increase antioxidant efficiencies via enzymatic and non-enzymatic antioxidants and the results are similar to previous reports that PBZ enhances antioxidant defence system by increasing the activities of SOD, CAT, APX, and POX and the contents of ascorbate, glutathione, and $\alpha$ tocopherol ${ }^{12-14}$. Enhancing antioxidant efficiency in plants by PBZ has also been reported in Catharanthus roseus ${ }^{12}$, barley ${ }^{27}$, and wheat ${ }^{34}$. PBZ also significantly prevented the degradation of photosynthetic pigments such as $\mathrm{Chl}_{\mathrm{a}}, \mathrm{Chl}_{\mathrm{b}}, \mathrm{TC}$, and $\mathrm{C}_{\mathrm{x}+\mathrm{c}}$ under salt stress (Table 3). It is probable that high antioxidant efficiencies may prevent degradation of chlorophylls and carotenoids due to their higher ability to scavenge and trap ROS before damaging cells.
In this experiment, PBZ pretreatment significantly enhanced POX and PPO activities under a normal condition (Figs. 4, 5). It has been reported that PBZ acts as a triazole fungicide ${ }^{11}$. Consequently, PBZ might induce ROS accumulation which is a signal molecule in the early period to induce the up-regulation of antioxidant enzyme. POX and PPO activities in leaves and roots of PTT1 rice seedlings under salt stress slightly increased for the first 3 days and decreased thereafter (Figs. 4, 5), suggesting that at the first stage of exposure to salinity, POX up-regulated might reduce the damage of cells and tissues by reducing toxic levels of $\mathrm{H}_{2} \mathrm{O}_{2}$ produced under stress ${ }^{34}$. PPO up-regulated might reduce degradation of the toxic substances such as phenolic compounds which are generally accumulated during stress condition, that lead to protecting cell from oxidative damage ${ }^{35}$. However, the prolonged period of salinity caused a decrease in POX and PPO activities, indicating that the ability to scavenge ROS by the enzymes was weakened leading to the overproduction of ROS and the degradation of photosynthetic pigments and finally in growth reduction. The decrease in POX or PPO activities due to $\mathrm{NaCl}$ salinity and the reduction of growth were reported in black gram ${ }^{36}$ and Withania somnifera ${ }^{37}$. In comparison, POX and PPO activities in the roots were higher than those in the leaves and slightly changed under salinity stress compared with the leaves (Figs. 4, 5) indicating that roots showed higher ROS scavenging by POX and PPO than leaves in a salinity condition. PBZ application greatly increased and restored POX and PPO activity in leaves and roots of PTT1 rice seedlings under salinity condition (Figs. 4, 5). It is probable that the increased POX and PPO activities could contribute to the antioxidant mechanism of PTT1 rice seedlings against salinity stress. Similar results were reported in cowpea ${ }^{13}$ and barley ${ }^{27}$. The increase in POX and PPO activities is assumed to be an adaptive trait for helping to overcome the toxic levels of ROS ${ }^{34,35}$.

\section{CONCLUSIONS}

This study demonstrated that salinity stress caused a reduction in growth performance, photosynthetic pigment and antioxidant efficiency. PBZ pretreatment effectively protected rice seedlings from salt stress by enhancing growth performance, photosynthetic pigment and antioxidant efficiency. This represents the ability of PBZ to repair the salt-induced damage in rice seedlings. Hence exogenous PBZ application might be a useful method to increase salt tolerant abilities in PTT1 rice. 
Acknowledgements: This study was financially supported by a grant from Thailand Graduate Institute of Science and Technology (TGIST, grant number TG-22-1055-005D); Faculty of Science and the Graduate School, Chiang Mai University, Chiang Mai, Thailand.

\section{REFERENCES}

1. Rengasamy P (2006) World salinization with emphasis on Australia. $J$ Exp Bot 57, 1017-23.

2. Munns R, Tester M (2008) Mechanisms of salinity tolerance. Annu Rev Plant Biol 59, 651-81.

3. Cha-um S, Kirdmanee C (2009) Effect of salt stress on proline accumulation, photosynthetic ability and growth characters in two maize cultivars. Pakistan $J$ Bot 41, 87-98.

4. Cha-um S, Kirdmanee C (2009) Proline accumulation, photosynthetic abilities and growth characters of sugarcane (Saccharum officinarum L.) plantlets in response to iso-osmotic salt and water-deficit stress. Agr Sci China 8, 51-8.

5. Chutipaijit S, Cha-um S, Sompornpailin K (2011) High contents of proline and anthocyanin increase protective response to salinity in Oryza sativa L. spp. indica. Aust J Crop Sci 5, 1191-8.

6. Sharma P, Jha AB, Dubey RS, Pessarakli M (2012) Reactive oxygen species, oxidative damage, and antioxidative defense mechanism in plants under stressful conditions. $J$ Bot 2012, Article ID 217037.

7. Gill SS, Tuteja N (2010) Reactive oxygen species and antioxidant machinery in abiotic stress tolerance in crop plants. Plant Physiol Biochem 48, 909-30.

8. Sharma I, Ching E, Saini S, Bhardwaj R, Pati PK (2013) Exogenous application of brassinosteroid offers tolerance to salinity by altering stress responses in rice variety Pusa Basmati-1. Plant Physiol Biochem 69, 17-26.

9. Rady MM, Mohamed GF (2015) Modulation of salt stress effects on the growth, physio-chemical attributes and yields of Phaseolus vulgaris L. plants by the combined application of salicylic acid and Moringa oleifera leaf extract. Sci Hort 193, 105-13.

10. Roychoudhury A, Basu S, Sengupta DN (2011) Amelioration of salinity stress by exogenously applied spermidine or spermine in three varieties of indica rice differing in their level of salt tolerance. $J$ Plant Physiol 168, 317-28.

11. Fletcher RA, Gilley A, Sankhla N, Davis TD (1999) Triazoles as plant growth regulators and stress protectants. In: Janick $\mathrm{J}$ (ed) Horticultural Reviews vol 24, pp 55-138., Wiley, Oxford.

12. Jaleel CA, Gopi R, Manivannan P, Panneerselvam $R$ (2007) Responses of antioxidant defense system of Catharanthus roseus (L.) G. Don. to paclobutrazol treatment under salinity. Acta Physiol Plant 29, 205-9.

13. Manivannan P, Jaleel CA, Kishorekumar A, Sankar B,
Somasundaram R, Panneerselvam R (2008) Protection of Vigna unguiculata (L.) Walp. plants from salt stress by paclobutrazol. Colloid Surf B 61, 315-8.

14. Sankar B, Jaleel CA, Manivannan P, Kishorekumar A, Somasundaram R, Panneerselvam R (2007) Effect of paclobutrazol on water stress amelioration through antioxidants and free radical scavenging enzymes in Arachis hypogaea L. Colloid Surf B 60, 229-35.

15. Lin KH, Tsou CC, Hwang SY, Chen LF, Lo HF (2006) Paclobutrazol pre-treatment enhanced flooding tolerance of sweet potato. J Plant Physiol 163, 750-60.

16. Cha-um S, Vejchasarn P, Kirdmanee C (2007) An effective defensive response in Thai aromatic rice varieties (Oryza sativa L. spp. indica) to salinity. J Crop Sci Biotechnol 10, 257-64.

17. Arnon DI (1949) Copper enzymes in isolated chloroplasts, polyphenol oxidase in Beta vulgaris. Plant Physiol 24, 1-15.

18. Huang D, Ou B, Prior RL (2005) The chemistry behind antioxidant capacity assays. J Agr Food Chem 53, 1841-56.

19. Mun'im A, Negishi O, Ozawa T (2003) Antioxidative compounds from Crotalaria sessiliflora. Biosci Biotechnol Biochem 67, 410-4.

20. Benzie IF, Strain JJ (1996) The ferric reducing ability of plasma (FRAP) as a measure of "antioxidant power": the FRAP assay. Anal Biochem 239, 70-6.

21. Huang S, Hart H, Lee H, Wicker L (1990) Enzymatic and color changes during post-harvest storage of lychee fruit. $J$ Food Sci 55, 1762-3.

22. Nagle NE, Haard NF (1975) Fractionation and characterization of peroxidase from ripe banana fruit. J Food Sci 40, 576-9.

23. Jiang Y, Fu J (1998) Inhibition of polyphenol oxidase and the browning control of litchi fruit by glutathione and citric acid. Food Chem 62, 49-52.

24. Lowry $\mathrm{OH}$, Rosebrough $\mathrm{NJ}$, Farr AL, Randall RJ (1951) Protein measurement with the Folin phenol reagent. $J$ Biol Chem 193, 265-75.

25. Geilfus CM, Zorb C, Muhling KH (2010) Salt stress differentially affects growth-mediating betaexpansins in resistant and sensitive maize (Zea mays L.). Plant Physiol Biochem 48, 993-8.

26. Boriboonkaset T, Theerawitaya C, Pichakum A, Chaum S, Takabe T, Kirdmanee C (2012) Expression levels of some starch metabolism related genes in flag leaf of two contrasting rice genotypes exposed to salt stress. Aust J Crop Sci 6, 1579-86.

27. Özmen AD, Özdemír F, Türkan I (2003) Effects of paclobutrazol on response of two barley cultivars to salt stress. Biol Plant 46, 263-8.

28. Hajihashemi S, Kiarostami K, Enteshari S, Saboora A (2006) The effects of salt stress and paclobutrazol on some physiological parameters of two salt tolerant and salt sensitive cultivars of wheat. Pakistan J Biol Sci 9, 1370-4.

29. Hajihashemi S, Kiarostami K, Saboora A, Enteshari 
S (2007) Exogenously applied paclobutrazol modulates growth in salt-stressed wheat plants. Plant Growth Regul 53, 117-28.

30. Wootton-Beard PC, Moran A, Ryan L (2011) Stability of the total antioxidant capacity and total polyphenol content of 23 commercially available vegetable juices before and after in vitro digestion measured by FRAP, DPPH, ABTS and Folin-Ciocalteu methods. Food Res Int 44, 217-24.

31. Li JT, Qiu ZB, Zhang XW, Wang LS (2011) Exogenous hydrogen peroxide can enhance tolerance of wheat seedlings to salt stress. Acta Physiol Plant 33, 835-42.

32. Sharma P, Dubey R (2005) Drought induces oxidative stress and enhances the activities of antioxidant enzymes in growing rice seedlings. Plant Growth Regul 46, 209-21.

33. Bian S, Jiang Y (2009) Reactive oxygen species, antioxidant enzyme activities and gene expression patterns in leaves and roots of Kentucky bluegrass in response to drought stress and recovery. Sci Hort 120, 264-70.

34. Kraus TE, McKersie BD, Fletcher RA (1995) Paclobutrazol-induced tolerance of wheat leaves to paraquat may involve increased antioxidant enzyme activity. J Plant Physiol 145, 570-6.

35. Weisany W, Sohrabi Y, Heidari G, Siosemardeh A, Ghassemi-Golezani K (2012) Changes in antioxidant enzymes activity and plant performance by salinity stress and zinc application in soybean (Glycine max L.). Plant Omics 5, 60-7.

36. Dash M, Panda SK (2001) Salt stress induced changes in growth and enzyme activities in germinating Phaseolus mungo seeds. Biol Plant 44, 587-9.

37. Jaleel CA, Lakshmanan GMA, Gomathinayagam M, Panneerselvam R (2008) Triadimefon induced salt stress tolerance in Withania somnifera and its relationship to antioxidant defense system. $S$ Afr $J$ Bot 74, 126-32. 